\title{
Two-Round Diagnosability Measures for Multiprocessor Systems
}

\author{
Jiarong Liang $\mathbb{D}^{1}{ }^{1}$ Qian Zhang, ${ }^{1}$ and Changzhen $\operatorname{Li} \mathbb{D}^{2}$ \\ ${ }^{1}$ School of Computer, Electronics and Information, and with Guangxi Key Laboratory of Multimedia \\ Communications and Network Technology, Guangxi University, Nanning 530004, China \\ ${ }^{2}$ School of Public Policy and Management, Guangxi University, Nanning 530004, China \\ Correspondence should be addressed to Changzhen Li; 19960126@gxu.edu.cn
}

Received 19 April 2020; Accepted 19 May 2020; Published 24 June 2020

Academic Editor: Shuping He

Copyright (C) 2020 Jiarong Liang et al. This is an open access article distributed under the Creative Commons Attribution License, which permits unrestricted use, distribution, and reproduction in any medium, provided the original work is properly cited.

\begin{abstract}
In a multiprocessor system, as a key measure index for evaluating its reliability, diagnosability has attracted lots of attentions. Traditional diagnosability and conditional diagnosability have already been widely discussed. However, the existing diagnosability measures are not sufficiently comprehensive to address a large number of faulty nodes in a system. This article introduces a novel concept of diagnosability, called two-round diagnosability, which means that all faulty nodes can be identified by at most a oneround replacement (repairing the faulty nodes). The characterization of two-round $t$-diagnosable systems is provided; moreover, several important properties are also presented. Based on the abovementioned theories, for the $n$-dimensional hypercube $\left(Q_{n}\right)$, we show that its two-round diagnosability is $\left(n^{2}+n / 2\right)$, which is $(n+1 / 2)$ times its classic diagnosability. Furthermore, a fault diagnosis algorithm is proposed to identify each node in the system under the PMC model. For $Q_{n}$, we prove that the proposed algorithm is the time complexity of $O\left(n 2^{n}\right)$.
\end{abstract}

\section{Introduction}

With the growth of the large scale integration technology, a huge number of multiprocessors are integrated to a multiprocessor computer system. It is not difficult to predict that, in such a system, some faulty processors (nodes) will be produced. To make sure that the system works properly, the designers should consider the problem that the system needs to have the ability to diagnose itself faulty processors such that they can be repaired or replaced with the new fault-free processors. In dealing with the problem of fault diagnosis for multiprocessor systems, two approaches are used: one is the system-level approach and another is the logic-circuit-level approach. Since the system-level approach is helpful for user-transparent reconfiguration, automatic, and recovery in the multiprocessor system while the logic-circuit approach is not, the designers prefer to design the system into a system-level fault-diagnosis system. In 1967, Preparata et al. proposed an automatic diagnosis procedure in multiprocessor systems, which is known as the first system-level diagnosis approach $[1,2]$. This model proposed by Preparata et al. [1] is called the Preparata, Metze, and Chien (PMC) model. In theory, a digraph $G=(V, E)$ can usually be used to denote a PMC model, where for two processors $i$ and $j$, $(i, j) \in E$ if and only if processor $u$ is tested processor $i$. For each testing edge $(i, j)$, we can use 1 or 0 to denote their test result $\sigma(i, j)$, where $\sigma(i, j)=1$ implies that $i$ judges $j$ to be faulty and $\sigma(i, j)=0$ implies that $i$ judges $j$ to be fault-free.

There are several fundamental system-level diagnosis strategies: $t$-diagnosis, $t / s$-diagnosis $(s \geq t)$ and conditional $t$-diagnosis. Suppose that a system $S$ has at most $t$ faulty nodes, if each node in the system can be diagnosed correctly as either fault-free or faulty, then the system is called a $t$-diagnosable system; some research results on a $t$-diagnosable system can be found in [1-4], etc. In theory, the $t$-diagnosis approach is a key measurement for the reliability of considered network system. Besides, the $t$-diagnosis approach is desirable to be applied to the areas being related to network control, for example, in the research on reinforcement learning and adaptive optimization, we know that the neural network is often used to represent actor network and chosen as a optimal control policy [5]. Before an adaptive optimal controller is designed, it is necessary and important to test whether the nodes (neurons) in the neural network are fault-free or faulty by the $t$-diagnosis approach. For a system having at most $t$ faulty nodes, if it can 
determine a set with the size $s(s \leq t)$ that contains all its faulty nodes, then it is $t / s$-diagnosable. Numerous studies have been reported on a $t / s$-diagnosable system, such as [6-12].

In a system, denoted by $G=(V, E)$, with at most $t$ faulty nodes, a subset $V_{i} \subset V$ with $\left|V_{i}\right| \leq t$ is called a conditional faulty set if there does not exist a node $v$ such that $N(v) \subset V_{i}$, where $N(v)$ is a set consisting of all $v / s$ neighbors. If for any two conditional faulty sets $V_{1}$ and $V_{2}$ with $V_{1} \neq V_{2}$, $\sigma\left(V_{1}\right) \cap \sigma\left(V_{2}\right)=\phi$, where $\sigma\left(V_{i}\right)$ is the set of syndromes produced by $V_{i}$, then the system is called conditionally $t$-diagnosable. Lots of efforts are made to study the conditionally $t$-diagnosable system, see [13-22].

It is worth mentioning that the above three strategies are oneround diagnosis strategies, whose diagnosabilities are usually not too large. For instance, $Q_{n}$ is shown to be $n$-diagnosable and its $t / t$-diagnosability and conditional diagnosability are $n$ and $(2 n-2) /(2 n-2)$, respectively, based on the PMC model. However, when the size of the faulty node set is larger than the diagnosability of the above diagnosis strategies, the above diagnosable systems can do little for the diagnosis. Therefore, to address the issue that a system has a large number of faulty nodes, Chen et al. introduced a novel strategy, called by $t / k$-diagnosis, for the star network [23], where $1 \leq k \leq t$. A $t / k$-diagnosable system guarantees to identify at least $k$ faulty nodes only if as long as the size of the set consisting of faulty nodes in it does not exceed $t$. Although a $t / k$-diagnosis has a large diagnosability, it takes much longer to repair faulty nodes, which leads to low efficiency. Therefore, it provides strong motivation for the study of a diagnosis strategy that can reach a balance between improving the diagnosability and being highly efficient. This paper presents a novel diagnosis approach, called by two-round $t$-diagnosis. Using the two-round $t$-diagnosis approach, the system can guarantee that each faulty node can be diagnosed by at most a one-round replacement (repairing the faulty nodes).

A simple introduction on this paper's remainder is as follows. Some related notations and definitions are presented as the preliminaries in Section 2. In Section 3, two-round $t$-diagnosable systems are characterized and several important properties are also presented. In Section 4, the properties of a two-round $t$-diagnosable system is applied for computing the two-round $t$-diagnosability of $Q_{n}$. In Section 5, a fast tworound diagnosis algorithm, whose time complexity is $O\left(n 2^{n}\right)$, is proposed for $Q_{n}$. Section 6 draws the conclusions.

\section{Preliminaries}

In the section, we introduce some necessary notations and definitions that are frequently used in the rest of the paper.

Under the PMC model, for a system given by graph $G=(V, E), \quad$ let $\Gamma x=\{y \mid(x, y) \in E$ and $x, y \in V\}$ and $\Gamma^{-1} x=\{y \mid(y, x) \in E$ and $x, y \in V\}$. Similarly, for any subset $X \subset V, \Gamma X=\cup_{x \in X} \Gamma x-X$ and $\Gamma^{-1} X=\cup_{x \in X} \Gamma^{-1} x-X$. In particular, if $G$ is undirected, then $\Gamma x=\Gamma x^{-1}=N(x)$ and $\Gamma X=\Gamma X^{-1}=N(X)$, where $X \subset V$.

Definition 1. Suppose that $G=(V, E)$ is a graph and has $k$ connected components, say $C_{1}, C_{2}, \ldots, C_{k}$. Then, $C_{\text {sub }}(G)=$ $\left\{C_{1}, C_{2}, \ldots, C_{k}\right\}$ is called a connected subgraph set of $G$. In particular, if $G$ is connected, then $C_{\text {sub }}(G)=\{G\}$.
Additionally, let $\max \left\{C_{\text {sub }}(G)\right\}=C_{i}$, where $\left|V\left(C_{i}\right)\right| \geq\left|V\left(C_{j}\right)\right|$ for each $C_{j} \in C_{\text {sub }}(G)$.

Definition 2. Let $G=(V, E)$ denote a graph and $X \subset V$. Then, $G[X]=(X, \bar{E})$ is called the induced subgraph by $X$, where $\bar{E}=\{(u, v) \mid(u, v) \in E, u, v \in X\}$.

Definition 3. Let $G=(V, E)$ denote a graph, let $\operatorname{Card}_{k}\left(C_{\text {sub }}(G)\right)=\left\{C \mid C \in C_{\text {sub }}(G)\right.$ and $\left.|V(C)|=k\right\}$ be the set of connected subgraphs with $k$ nodes in $G$.

For instance, for graph $G$ shown in Figure $1, C_{\text {sub }}(G)=$ $\left\{C_{1}, C_{2}\right\}$, where $C_{1}=G\left[\left\{v_{2}, v_{3}, v_{6}\right\}\right], C_{2}=G\left[\left\{v_{1}, v_{4}, v_{5}, v_{7}\right\}\right]$, $\operatorname{Card}_{3}\left(C_{\text {sub }}(G)\right)=G\left[\left\{v_{2}, v_{3}, v_{6}\right\}\right]$, and $\operatorname{Card}_{4}\left(C_{\text {sub }}(G)\right)=$ $G\left[\left\{v_{1}, v_{4}, v_{5}, v_{7}\right\}\right]$.

Definition 4. Let $G=(V, E)$ denote a system, $M \subset V$. For a given syndrome $\sigma$, if the following conditions are satisfied, then $M \subset V$ is said to be an allowable fault set (AFS):

(1) $\sigma(x, y)=0$, for any $x, y \in V-M$

(2) $\sigma(x, y)=1$, for any $x \in V-M$ and $y \in M$

Lemma 1. Suppose that $G=(V, E)$ is a system and $\sigma$ is a given syndrome. Let $F_{1}, F_{2} \subseteq V$ be two AFSs, then $F_{1} \cup F_{2}$ is also an AFS.

Proof. To the contrary, assume that $F_{1} \cup F_{2}$ is not an AFS; then, there exists at least one condition in Definition 4, which is not true.

If condition (1) is not true, then $\exists x, y \in V-\left(F_{1} \cup F_{2}\right)$ such that $\sigma(x, y)=1$, a condition to $F_{1}$ and $F_{2}$ are two AFS.

If condition (2) is not true, then $\exists x \in V-\left(F_{1} \cup F_{2}\right)$ and $y \in F_{1} \cup F_{2}$, where $(x, y) \in E$ such that $\sigma(x, y)=0$. If $y \in F_{1}$, then $F_{1}$ is not an AFS; if $y \in F_{2}$, then $F_{2}$ is not an AFS, which contradicts the hypothesis.

Lemma 2. For a given syndrome $\sigma$ on the system $G=(V, E)$, suppose that $G$ has $k$ AFSs for $\sigma$, say $F_{1}, F_{2}, \ldots, F_{k} \subseteq V$, then, $\cap_{i=1}^{k} F_{i}$ is the fault set of the system.

Proof. Let $F_{c}=\cap_{i=1}^{k} F_{i}$. Let $F$ be the maximal fault set, which exactly consists of all faulty nodes. We will show that $F$ is an AFS for $\sigma$. To this end, let $(u, v) \in E . u, v \in V-F$ implies that $u$ and $v$ are fault-free nodes, and then $\sigma(u, v)=0$. Hence, condition (1) holds for $F$. For condition (2), $u \in V-F$ and $v \in F$ implies that $u$ is a fault-free node and $v$ is a faulty node and then that $\sigma(x, y)=1$. Hence, condition (2) holds for $F$. Then, $F$ is an AFS for $\sigma$. According to the assumption, we have that $F \in\left\{F_{1}, F_{2}, \ldots, F_{k}\right\}$. So, $F_{c}=\cap_{i=1}^{k} F_{i} \subseteq F$.

Definition 5. Let $G=(V, E)$ be a system, $t$ an integer, $X \subseteq V$ with $0<|X|<t$. Suppose that $X_{1}, X_{2} \subseteq V-X$ with $\left|X_{1} \cup X\right| \leq t$ and $\left|X_{2} \cup X\right| \leq t$ are two different subsets. $\left(X_{1}, X_{2}\right)$ is called a pair of distinguishable subsets of $V-X$ if there exists an edge from $X$ to $X_{1} \Delta X_{2}$. 


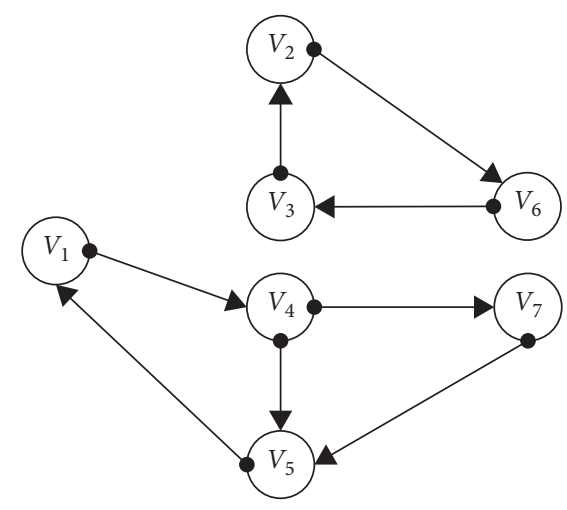

FIgURE 1: A graph with 7 nodes.

According to Definition 5, the following results are true. In the system $G=(V, E)$, if $X \subseteq V$ with $|X|<k$, any two subsets $X_{1} \subseteq V-X$ with $\left|X_{1} \cup X\right| \leq t, X_{2} \subseteq V-X$ with $\left|X_{2} \cup X\right| \leq t$, and $\left(X_{1}, X_{2}\right)$ is a pair of distinguishable subsets in $V-X$, then the system can determine the fault set, provided that $G$ has less than $k$ faulty nodes and all faulty nodes in $X$ have been repaired or replaced with additional fault-free nodes.

Lemma 3. Suppose that that the undirected graph $G=(V, E)$ has less than $t$ faulty nodes and $G \prime \subset G$ with $\left|V\left(G^{\prime}\right)\right| \geq t+1$ is connected. If each result in $G^{\prime}$ is 0 , then $G \prime$ does not have faulty nodes.

Proof. To the contrary, let $G^{\prime} \subset G$ with $\left|V\left(G^{\prime}\right)\right| \geq t+1$ be connected, in which each result in it is 0 and there is a faulty node, say $u$. Then, it is clear that each node in $N_{G^{\prime}}(u)$ is faulty. Similarly, each node in $N_{G^{\prime}}\left(N_{G^{\prime}}(u)\right)$ is 1 faulty. As a result, each node $V\left(G^{\prime}\right)$ is faulty. Note that $\left|V\left(G^{\prime}\right)\right| \geq t+1$; this implies that the number of fault nodes in $G^{\prime}$ exceeds $t$, a contradiction. Therefore, each node in $V\left(G^{\prime}\right)$ is fault-free.

\section{Two-Round $t$-Diagnosable Systems}

At the beginning of the section, the concept of a two-round $t$-diagnosable system is presented as follows.

Definition 6. A system is two-round $t$-diagnosable if, for given syndrome $\sigma$, after repairing or replacing the faulty nodes identified by one-round diagnosis, the system can diagnose the remaining faulty nodes without replacement, provided that the system has less than $t+1$ faulty nodes.

According to Definition 6, we can obtain the following necessary conditions for a two-round $t$-diagnosable system.

Theorem 1. Let $G=(V, E)$ represent a system. Then, $G$ is two-round $t$-diagnosable if and only if for any a subset $Y \subseteq V$ with $|Y| \leq t$ and any two distinct subsets $Y_{1}, Y_{2} \subseteq V-Y$, where $\left|Y \cup Y_{1}\right| \leq t,\left|Y \cup Y_{2}\right| \leq t$, and $Y_{1} \cap Y_{2}=\phi$, there exists at least an edge from $V-Y_{1}-Y_{2}$ to $Y_{1} \Delta Y_{2}$.

Proof.

Necessity: since for any $Y \subseteq V$ with $|Y|=t$, the result is trivial, next, we show that the result is true for the case of
$|Y|<t$. To the contrary, suppose that there exists a subset $Y \subseteq V$, where $|Y|<t$ and distinct subsets $Y_{1}, Y_{2} \subseteq V-Y$ with $\left|Y \cup Y_{1}\right| \leq t,\left|Y \cup Y_{2}\right| \leq t, Y_{1} \cap Y_{2}=\phi$, such that there are no edges from $V-Y_{1}-Y_{2}$ to $Y_{1} \Delta Y_{2}$. Without loss of generality, suppose that each node in $Y$ is faulty and $G$ has more than $|Y|$ faulty nodes. Define a syndrome $\sigma$ as follows. Let $x, y \in V$ with $(x, y) \in E$ :

(1) If $x, y \in V-Y-Y_{1}-Y_{2}$, then $\sigma(x, y)=0$

(2) If $x \in V-Y-Y_{1}-Y_{2}$ and $y \in Y$, then $\sigma(x, y)=1$

(3) If $x \in Y_{1}-Y_{2}$ and $y \in Y_{2}-Y_{1}$, then $\sigma(x, y)=1$

(4) If $x \in Y_{2}-Y_{1}$ and $y \in Y_{1}-Y_{2}$, then $\sigma(x, y)=1$

(5) The remaining test results are arbitrary

Both $\left(Y \cup Y_{1}\right)$ and $\left(Y \cup Y_{2}\right)$ are allowable sets for syndrome $\sigma$. Suppose that $F_{1}, F_{2}, \ldots, F_{k} \subseteq V$ are all the allowable fault sets for $\sigma$. Let $F_{c}=\cap_{i=1}^{k} F_{i} ; F_{c}$ is a fault set of $G$ and $F_{c} \subseteq Y$, which implies that the fault set $Y_{1}$ identified by the first-round diagnosis is a subset of $Y$. Let $G$ ' denote the system after replacing the nodes of $Y_{1}$, and $\sigma$ is a syndrome obtained by performing a test task on $G$ '. Since there are no edges from $V-Y_{1}-Y_{2}$ to $Y_{1} \Delta Y_{2}$, then $\sigma(x, y)=$ $\sigma(x, y)=1$ for $x \in Y_{1}-Y_{2}, y \in Y_{2}-Y_{1}$, and $\sigma(x, y)=$ $\sigma(x, y)=1$ for $x \in Y_{2}-Y_{1}, y \in Y_{1}-Y_{2}$. Hence, both $Y_{1}$ and $Y_{2}$ are AFSs for $\sigma$, which is a contradiction to the hypothesis that $G=(V, E)$ is two-round $t$-diagnosable.

Sufficiency: for a syndrome $\sigma$, let $F_{c}$ be the intersection of all AFSs for $\sigma$. According to Lemma 2, $F_{c}$ is a fault set, where $\left|F_{c}\right| \leq t$. If $\left|F_{c}\right|=t$, then each system has been diagnosed by syndrome $\sigma$, which implies that $G$ is tworound $t$-diagnosable. If $\left|F_{c}\right|<t$, then let $G$ ' denote the system for which all the nodes in $F_{c}$ are repaired or replaced with additional fault-free nodes from $G$. Then, the number of faulty nodes in $G$ ' will not be more than $t-\left|F_{c}\right|$, and these faulty nodes belong to $V-F_{c}$. Let $\sigma$ denote a syndrome obtained by performing the test task to $G \prime$. We claim that the fault set $Y_{1} \subseteq V-F_{c}$ of $G$ ' where $\left|Y_{1}\right| \leq t-\left|F_{c}\right|$ can be determined by $\sigma$. In contrast, there exists another nonempty allowable subset $Y_{2} \subseteq V-$ $F_{c}$ of $G^{\prime}$, where $\left|Y_{2}\right| \leq t-\left|F_{c}\right|$ for $\sigma$, and we derive a contradiction. Consider the following situations.

Case 1: there is an edge $(x, y)$ from $V-F_{c}-Y_{1}-Y_{2}$ to $Y_{1} \Delta Y_{2}$. Without loss of generality, let $y \in Y_{1}$. Since $Y_{2}$ is an allowable subset of $V-F_{c}$ for $\sigma, \sigma(x, y)=0$. On the contrary, since $Y_{1}$ is a fault subset of $V-F_{c}$ for $\sigma$, $\sigma(x, y)=1$ is a contradiction.

Case 2: there are no edges from $V-F_{c}-Y_{1}-Y_{2}$ to $Y_{1} \Delta Y_{2}$. According to this assumption, there exists an edge $(x, y)$ from $F_{c}$ to $Y_{1} \Delta Y_{2}$. Without loss of generality, suppose that $y \in Y_{1}-Y_{2}$. Since $Y_{2}$ is an allowable set for $\sigma, \sigma(x, y)=0$. On the contrary, since $Y_{1}$ is a fault set of $G^{\prime}, \sigma(x, y)=1$ is a contradiction. Hence, $G$ is two-round $t$-diagnosable.

According to the proof of Theorem 1, the two corollaries described as follows are obvious.

Corollary 1. A system $G=(V, E)$ is two-round t-diagnosable if for any subset $Y \subseteq V$ with $|Y|<t$, and for any distinct disjoint 
subsets $Y_{1}, Y_{2} \subseteq V-Y$, where $\left|Y \cup Y_{1}\right| \leq t$ and $\left|Y \cup Y_{2}\right| \leq t$, and $\left(Y_{1}, Y_{2}\right)$ is a pair of distinguishable subsets of $V-Y$.

Definition 7. Let $S$ be a network system. The maximum nonnegative integer $t$ that guarantees $S$ to be two-round $t$-diagnosable is called the two-round diagnosability of $S$.

For convenience, it is necessary to introduce a notation $\Gamma_{2}^{-1} v=\Gamma^{-1} v \cup \Gamma^{-1}\left(\Gamma^{-1} v\right)-\{v\}$ for the coming corollary, where $G=(V, E)$ is a system, $v \in V$.

Corollary 2. For the system $G=(V, E)$, let $\alpha=\min \left\{\left|\Gamma_{2}^{-1} v\right| \mid v \in V\right\}+1$. Then, the system is not tworound $\alpha$-diagnosable.

Proof. Let $v \in V$ be a node such that $\alpha=\left|\Gamma_{2}^{-1} v\right|+1$. Consider the case such that $F=\Gamma_{2}^{-1} v \cup\{v\}$ is a fault set that consists of exactly all faulty nodes in the system. Note that $|F|=\alpha$. Define a syndrome $\sigma$ as follows. Let $x, y \in V$ with $(x, y) \in E$ (see Figure 2):

(i) If $x, y \in V-\Gamma_{2}^{-1} v-\{v\}$, then $\sigma(x, y)=0$

(ii) If $x \in V-\Gamma_{2}^{-1} v-\{v\}$ and $y \in \Gamma^{-1}\left(\Gamma^{-1} v\right)-\{v\}$, then $\sigma(x, y)=1$ (before replacement)

(iii) If $x \in \Gamma^{-1}\left(\Gamma^{-1} v\right)-\{v\}$ and $y \in V-\Gamma_{2}^{-1} v-\{v\}$, then $\sigma(x, y)=0$

(iv) If $x \in \Gamma^{-1}\left(\Gamma^{-1} v\right)-\{v\}$ and $y \in \Gamma^{-1} v$, then $\sigma(x, y)=1$

(v) If $x \in \Gamma^{-1} v$, then $\sigma(v, x)=1$

(vi) If $x \in \Gamma^{-1}\left(\Gamma^{-1} v\right)-\{v\}$, then $\sigma(v, x)=0$

(vii) If $x \in \Gamma^{-1} v$, then $\sigma(v, x)=1$

(viii) If $x \in \Gamma^{-1}\left(\Gamma^{-1} v\right)-\{v\}$, then $\sigma(v, x)=1$ (before replacement)

(ix) The other test results are arbitrary

For $\sigma$, the nodes of subset $\Gamma^{-1}\left(\Gamma^{-1} v\right)-\{v\}$ can be identified correctly as faulty, which implies that the nodes of subset $\Gamma^{-1} v \cup\{v\}$ cannot be identified by syndrome $\sigma$. After the faulty nodes of subset $\Gamma^{-1}\left(\Gamma^{-1} v\right)-\{v\}$ are repaired, there exist no edges from fault-free nodes to $v$, and the test results from $v$ to faulty (fault-free) nodes are $1(0)$, which implies that we cannot judge the state of node $v$. Therefore, to identify the state of node $v$, we need a second replacement. So, the system is not two-round $\alpha$-diagnosable.

\section{Two-Round Diagnosability of Hypercube Networks}

$Q_{n}$ is a regular graph with $2^{n}$ nodes and $n 2^{n}$ edges. Each node in $Q_{n}$ can be denoted by an $n$-bit binary string. $(x, y) \in E\left(Q_{n}\right)$ if and only if there is exactly different one bit position between $x$ and $y$. Figure 3 is an illustration of a 4dimensional hypercube network $Q_{4}$.

Lemma 4 (see [17]). Let $X \subset V\left(Q_{n}\right)$ with $|Y|=s$ and $0>s \leq n+1$. Then, the size of the neighbor set of $Y$ is more than $(s n-s(s+1) / 2)$.

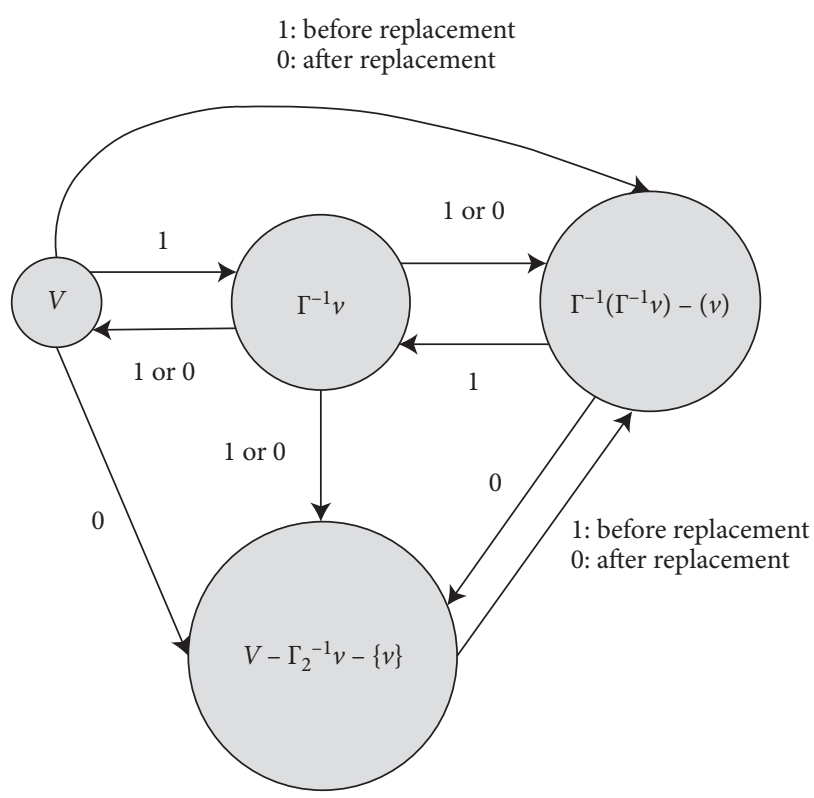

FIgURE 2: A syndrome of Corollary 2.

Lemma 5. For $Q_{n} \quad(n \geq 5)$, let $S=\left\{v_{i} \mid v_{i} \in V\left(Q_{n}\right)\right.$, $\left.1 \leq i \leq n,\left|\cap_{i=1}^{n} N\left(v_{i}\right)\right|=1\right\}$. Then, $|N(S)|=\left(n^{2}-n / 2\right)+1$.

Proof. We use add $(v)$ to denote $v^{\prime}$ address. Suppose that $\cap_{i=1}^{n} N\left(v_{i}\right)=\left\{v_{0}\right\}$ and add $\left(v_{0}\right)=a_{1} a_{2} \cdots a_{n}$. According to the definition of $Q_{n}$, without loss of generality, assume that $\operatorname{add}\left(v_{1}\right)=\overline{a_{1}} a_{2} \cdots a_{n}, \quad \operatorname{add}\left(v_{2}\right)=a_{1} \overline{a_{2}} \cdots a_{n}, \ldots, \operatorname{add}\left(v_{n}\right)=$ $a_{1} a_{2} \cdots \overline{a_{n}}$. For some $i$, we have that $N\left(v_{i}\right)=\left\{\overline{a_{1}} a_{2} \cdots \bar{a}\right.$ $\left.i \cdots a_{n}, a_{1} \overline{a_{2}} a_{3} \cdots \overline{a_{i}} \cdots a_{n}, \ldots, a_{1} a_{2} \cdots \overline{a_{i}} \ldots \overline{a_{n}}\right\}$. Then, $N\left(v_{i}\right) \cap N\left(v_{j}\right)=\left\{v_{0}, u\right\}$, where $\operatorname{add}(u)=a_{1} a_{2} \ldots \overline{a_{i}} \ldots$ $\overline{a_{j}} \cdots a_{n}$ and $1 \leq i, j \leq n, i \neq j$. So, $|N(S)|=n(n-1)-C_{n}{ }^{2}+$ $1=n^{2}-(n(n+1) / 2)+1=\left(n^{2}-n / 2\right)+1$.

According to Lemmas 4 and 5, for an $n$-dimensional hypercube and a subset $S \subseteq V$, where $|S|=n+1$, if $\exists v \in S$ with $N(v) \subseteq S$, then $|N(S)|=\left(n^{2}-n / 2\right)$.

Lemma 6 (see [19]). Suppose that $Q_{n}$ is modelled by a graph $G(V, E) \quad(n \geq 5)$. Let $S \subset V$ with $n \leq|S| \leq 2(n-1)-1$. If $G[V-S]$ is disconnected and $C_{\text {sub }}(G[V-S])=$ $\left\{C_{1}, C_{2}, \ldots, C_{m}\right\}$, then the following conditions hold:

(i) $\sum_{i=0}^{1} i\left|\operatorname{Card}_{i}\left(C_{\text {sub }}((G[V-S]))\right)\right| \leq 1$

(ii) There is unique $C_{i} \in C_{\text {sub }}(G[V-S])$, where $\left|V\left(C_{i}\right)\right| \geq 2$

Lemma 7 (see [24]). Suppose that $Q_{5}$ is modelled by a graph $G(V, E)$. Let $S \subset V$ with $\leq 5 k-(k(k+1) / 2) \quad(1 \leq k \leq 4)$. If $G[V-S]$ is disconnected and $C_{\text {sub }}(G[V-S])=$ $\left\{C_{1}, C_{2}, \ldots, C_{m}\right\}$, then the following conditions hold:

(i) $\sum_{i=0}^{k-1} i\left|\operatorname{Card}_{i}\left(C_{\text {sub }}(G[V-S])\right)\right| \leq k-1$

(ii) There is unique $C_{i} \in C_{\text {sub }}(G[V-S])$, where $\left|V\left(C_{i}\right)\right| \geq k$ 


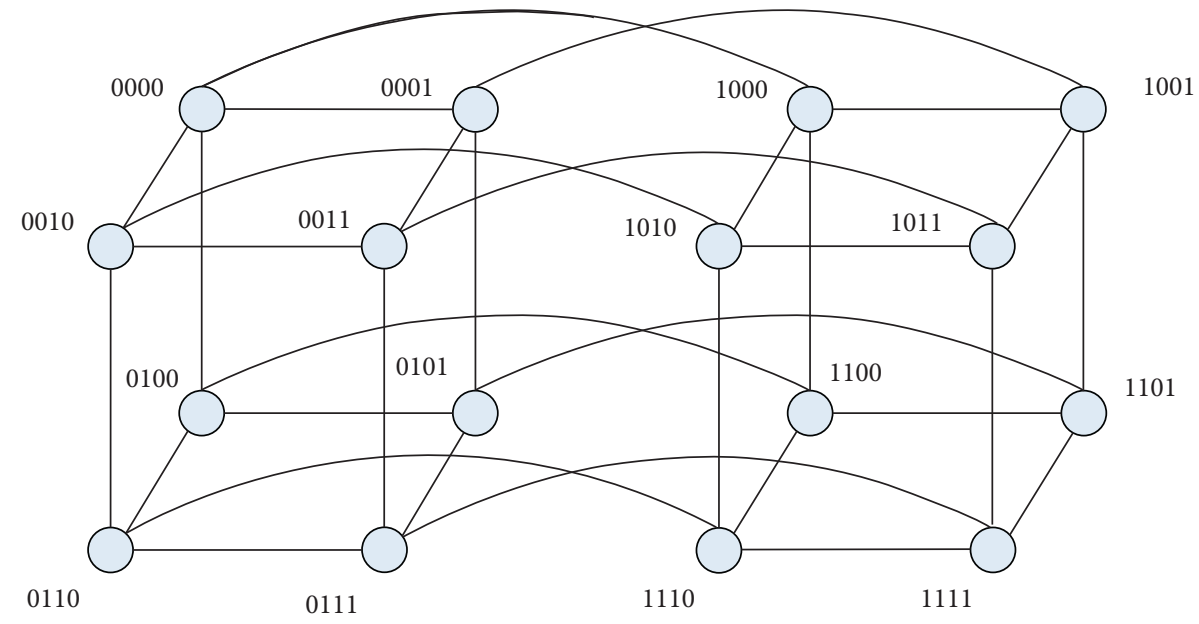

FIgURE 3: A 4-dimensional hypercube.

Lemma 8 Let $x>0$ and $y>0$ are two integers. Then, $(x+y) \leq 1+x y$.

Proof. Consider the function $g(x, y)=(1+x y)-(x+y)$. It is obvious that $g(x, y)=(x-1)(y-1)$. Since $x \geq 1$ and $y \geq 1$, then $g(x, y) \geq 0$, which implies that $(x+y) \leq 1+x y$.

Lemma 9 (see [24]). Suppose that $Q_{n}$ is modelled by a graph $G(V, E) \quad(n \geq 5)$. Let $S \subset V$ with $|S| \leq k n-(k(k+1) / 2)$ $(1 \leq k \leq n-1)$. If $G[V-S]$ is disconnected and $C_{\text {sub }}(G[V-S])=\left\{C_{1}, C_{2}, \ldots, C_{m}\right\}$, then the following conditions are true:

(i) $\sum_{i=0}^{k-1} i\left|\operatorname{Card}_{i}\left(C_{\text {sub }}(G[V-S])\right)\right| \leq k-1$

(ii) There is unique $C_{i} \in C_{\text {sub }}(G[V-S])$, where $\left|V\left(C_{i}\right)\right| \geq k$

Lemma 10 (see [24]). Suppose that $Q_{5}$ is modelled by a graph $G(V, E)$. Let $S \subset V$ with $|S| \leq 9$. If $G[V-S]$ is disconnected and $C_{\text {sub }}(G[V-S])=\left\{C_{1}, C_{2}, \ldots, C_{m}\right\}$, then the following conditions are true:

(i) $\sum_{i=0}^{k-1} i\left|\operatorname{Card}_{i}\left(C_{\text {sub }}(G[V-S])\right)\right| \leq 4$

(ii) There is unique $C_{i} \in C_{\text {sub }}(G[V-S])$, where $\left|V\left(C_{i}\right)\right| \geq 5$

Lemma 11 (see $[24,25])$. Suppose that $Q_{n}$ is modelled by a graph $G(V, E) \quad(n \geq 5)$. Let $S \subset V \quad$ with $|S| \leq n^{2}-(n(n+1) / 2)-1$. If $G[V-S]$ is disconnected and $C_{\text {sub }}(G[V-S])=\left\{C_{1}, C_{2}, \ldots, C_{m}\right\}$, then the two conditions as follows are true:

(i) $\sum_{i=0}^{n-1} i\left|\operatorname{Card}_{i}\left(C_{\text {sub }}(G[V-S])\right)\right| \leq n-2$

(ii) There is unique $C_{i} \in C_{\text {sub }}(G[V-S])$, where $\left|V\left(C_{i}\right)\right| \geq n$

With the above preliminaries, we shall discuss the tworound diagnosability of $Q_{n}$.
Theorem 2. An n-dimensional $(n \geq 6)$ hypercube given by $G=(V, E)$ is not two-round $\left(n^{2}+n / 2\right)+1$-diagnosable.

Proof. Note that, for each node $v \in V$, we have $\left|\Gamma_{2}^{-1} v \cup\{v\}\right|=$ $\left(n^{2}+n / 2\right)+1$ by Lemma 5 . According to Corollary 2, it is easily determined that an $n$-dimensional hypercube is not two-round $\left(n^{2}+n / 2\right)+1$-diagnosable.

Theorem 3. An $n$-dimensional $(n \geq 6)$ hypercube given by $G=(V, E)$ is two-round $\left(n^{2}+n / 2\right)$-diagnosable.

Proof. Let $X \subseteq V$ be a subset of $V$, where $|X|<\left(n^{2}+n / 2\right)$. According to Theorem 1, we will show that, for any two distinct subsets $S_{1}, S_{2} \subseteq V-X$, where $\left|X \cup S_{1}\right| \leq\left(n^{2}+n / 2\right)$, $\left|X \cup S_{2}\right| \leq\left(n^{2}+n / 2\right)$, and $S_{1} \cap S_{2}=\phi$, there exists at least an edge from $V-S_{1}-S_{2}$ to $S_{1} \Delta S_{2}$. Without loss of generality, let $S_{1}-S_{2} \neq \phi$.

Now, consider the two situations:

Case 1: $|X| \geq\left(n^{2}-n / 2\right)$.

Note that $\left|S_{1}\right|<\left(n^{2}+n / 2\right)-|X| \leq\left(n^{2}+n / 2\right)-$ $\left(n^{2}-n / 2\right)=n$ and $\left|S_{2}\right|<\left(n^{2}+n / 2\right)-|X| \leq\left(n^{2}+n / 2\right)-$ $\left(n^{2}-n / 2\right)=n$, which implies that $\left|\left(S_{1}-S_{2}\right)\right|<n$. Let $k=\left|\left(S_{1}-S_{2}\right)\right| ;$ then, $1 \leq k<n$. According to Lemma 4, we have that $N\left(S_{1}-S_{2}\right) \geq k n-(k(k+1) / 2)+1$. Since $f(k)=k n-(k(k+1) / 2)+1$ is an increasing function for $k \in[1, n-(1 / 2)], f(k) \geq f(1)=n$, which implies that $\left|S_{1}-S_{2}\right|=f(k)>n>\left|S_{2}\right|$. Hence, there exists at least an edge from $V-S_{1}-S_{2}$ to $S_{1}-S_{2}$.

Case 2: $|X|<\left(n^{2}-n / 2\right)$.

To the contrary, assume that $\exists S_{1}, S_{2} \subseteq V-X$ with $\left|X \cup S_{1}\right| \leq\left(n^{2}+n / 2\right),\left|X \cup S_{2}\right| \leq\left(n^{2}+n / 2\right), \quad$ and $S_{1} \cap S_{2}=\phi$, but that there are no edges from $V-S_{1}-S_{2}$ to $S_{1} \Delta S_{2}$. Next, we derive a contradiction. According to Lemma 11, we know that the system can be divided into three parts by a subset $X$ (shown in Figure 4). Note that $A$ is the largest component of $G[V-X]$, where $|A| \geq 2^{n}-\left(\left(n^{2}-n / 2\right)-1\right)-(n-1)=2^{n}+2-\left(n^{2}+n / 2\right)$, and $B$ is the union of the remaining components of $G[V-X]$, where $|B| \leq n-1$. Since $|B| \leq n-1$ and $Q_{n}$ is a 


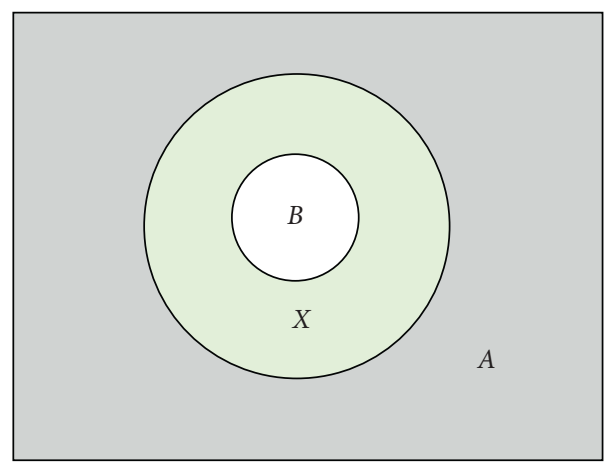

Figure 4: System divided into $A, B$, and $X$ by subset $X$.

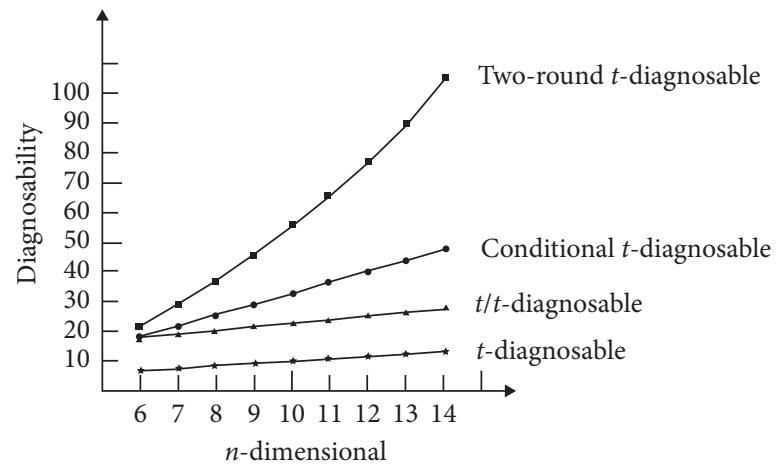

Figure 5: Comparison of the diagnosabilities of $Q_{n}$ between the traditional diagnosis approach and the two-round diagnosis approach.

$n$-regular graph, $S_{1} \cap B=\phi$. A similar argument can be used to obtain that $S_{2} \cap B=\phi$. Hence, $S_{1} \subseteq A$, and $S_{2} \subseteq A$.

Note that $\left|A-S_{1} \cup S_{2}\right|=|A|-\left|S_{1}\right|-\left|S_{2}\right| \geq 2^{n}-|X|-$ $\left|S_{1}\right|-\left|S_{2}\right|-(n-1) \geq 2^{n}-(3 / 2)\left(n^{2}+n / 2\right)-(n-1)>0$ $(n \geq 6)$, and $A$ is a component of $V-S$. This property implies that $N\left(S_{1} \Delta S_{2}\right) \cap A \neq \phi$, which is a contradiction.

In summary, we conclude that $Q_{n}(n \geq 6)$ is two-round $\left(n^{2}+n / 2\right)$-diagnosable.

As we know, $Q_{n}$ is $n$-diagnosable, $(2 n-2 / 2 n-2)$-diagnosable, and conditionally $4 n-7$-diagnosable. A previous study has shown that $Q_{n}$ is also two-round $\left(n^{2}+n / 2\right)$-diagnosable. Figure 5 gives an intuitive comparison between these diagnosabilities for $Q_{n}$.

\section{A Fault Diagnosis Algorithm of Two Round t-Diagnosable Hypercubes}

In Section 4 , we observed that an $n$-dimensional hypercube $\left(Q_{n}\right)$ was two-round $\left(n^{2}+n / 2\right)$-diagnosable. Then, identifying all faults with at most a one-round replacement remains an open question. This section presents a fast identification algorithm to address this issue (Algorithm 1. The completeness of the identification algorithm is demonstrated, provided that the system has less than $t+1$ faulty nodes $\left(t \leq\left(n^{2}-n / 2\right)-1\right)$. The fast identification algorithm is described in detail in Algorithm 2.
Algorithm 1 is applied to each node of $Q_{n}$ with at most $t$ faulty nodes $\left(t \leq\left(n^{2}-n / 2\right)-1\right)$. According to Lemmas 9 and 11, the unique set $S$ can be output by Algorithm DFS, where $|S| \geq t+1$.

Theorem 4. The time complexity of the fast identification algorithm is $\mathrm{O}\left(\mathrm{N} \log _{2} N\right)$, where $N=2^{n}$.

Proof. For the sake of convenience, let $F$ denote a set exactly consisting of faulty nodes in the system, $L$ the largest component of the induced subgraph by $V-F$, and $S$ a set consisting of all remaining small components in the induced subgraph by $V-F$. When $\left.u_{i} \in L\right)$, Step 1 takes an amount of time equal to $O\left(N \log _{2} N\right)$. When the $\left(u_{i}\right) \in S \cup F$, Step 1 takes an amount of time equal to $O\left(\left(n^{2}+n / 2\right)+N \log _{2} N\right)=O\left(N \log _{2} N\right)$. Hence, Step 1 takes an amount of time equal to $O\left(N \log _{2} N\right)$. Step 2 and Step 3 take an amount of time equal to $O(N)$. So, the total time for Fast Identification is $\mathrm{O}\left(\mathrm{N} \log _{2} \mathrm{~N}\right)$.

According to Lemmas 9 and 11, the completeness of the fast identification algorithm is obvious, provided that $Q_{n}$ has no more than $\left(n^{2}-n / 2\right)-1$ faulty nodes. Note that $Q_{n}$ is two-round $\left(n^{2}+n / 2\right)$-diagnosable. Then, there is a question of whether our algorithm is suitable for the scenario in which there are $t\left(\left(n^{2}-n / 2\right) \leq t \leq\left(n^{2}+n / 2\right)\right)$ faults in the system. We perform a simulation to evaluate the system; in the following simulation, we assume that $Q_{n}$ has $\left(n^{2}+n / 2\right)$ 


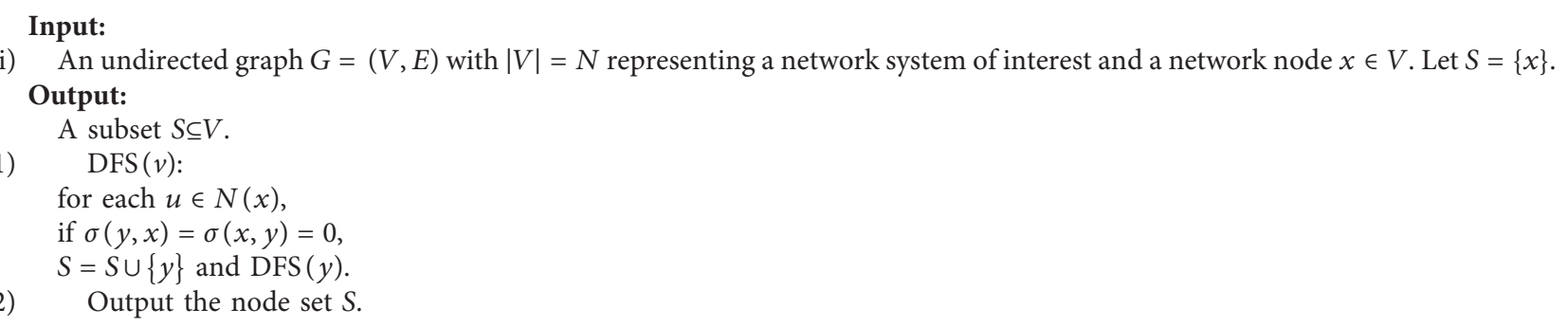

Algorithm 1: Depth-first search.

Input:

An undirected graph $G=(V, E)$ with $|V|=2^{n}$, a positive integer $t\left(t \leq\left(n^{2}-n / 2\right)-1\right)$ and a syndrome $\sigma$.

(i) Output:

A fault set $A$, a fault-free node set $B$ and a second-round fault set $C$, where $A \cup B \cup C=V$.

(1) Let $S_{i}=\varnothing\left(1 \leq i \leq 2^{n}\right)$ and $B=A=C=\varnothing$.

For any $u_{i} \in V-\cup_{j=1}^{i} S_{i}\left(1 \leq i \leq 2^{n}\right)$, perform $\operatorname{DAS}\left(u_{i}\right) ; S_{i} \operatorname{DAS}\left(u_{i}\right)$.

while $\left|S_{i}\right| \geq t+1$, let $B=B \cup S_{i}$, and $A=A \cup N(B)$.

(2) While $V=B \cup A$, output $B$ and $A$. Otherwise, go to Step 3.

(3) While $|A| \geq t$, let $B=V-A$, output $B$ and $A$.

Otherwise, repair the faulty nodes in $A$ and execute

Best $(A, B, C)$ until $B \cup A \cup C=V$. Additionally, output subsets $B, A$, and $C$.

Best $(A, B, C)$ :

For any $v \in V-A-B$, if $\sigma(u, v)=1(u \in A \cup B)$, then $C=C \cup\{v\}$; otherwise, $B=B \cup\{v\}$.

Algorithm 2: Fast identification.

TABLE 1: Number of faulty nodes identified by the two-round algorithm applied to an $n$-dimensional hypercube.

\begin{tabular}{lcccccc}
\hline$n$ & 7 & 8 & 9 & 10 & 11 & \\
\hline Faults & 28 & 36 & 45 & 66 & 78 & 91 \\
Identified faults & 28 & 36 & 45 & 66 & 78 & 91 \\
\hline
\end{tabular}

faulty nodes, and each node of the system may be faulty with the same probability. We run our algorithm 1000 times. Table 1 gives the corresponding experimental results.

The simulation shows that our algorithm is suitable for $Q_{n}$, provided that it has no more than $\left(n^{2}+n / 2\right)$ faulty nodes.

\section{Conclusion}

In this article, we introduce a novel diagnosis strategy called the two-round diagnosis strategy that implies that each node can be determined by at most a one-round replacement. A necessary and sufficient condition of the system being tworound $t$-diagnosable is presented. Additionally, several important properties of this system are described. Using the theory of a two-round $t$-diagnosable system, we show that $Q_{n}$ is two-round $\left(n^{2}+n / 2\right)$-diagnosable. Compared to the traditional diagnosis strategy, the two-round diagnosability of $Q_{n}$ is $(n+1 / 2)$ times as large as $n$, the classic diagnosability of $Q_{n}$. Furthermore, an $O\left(n 2^{n}\right)$ algorithm is provided to identify faulty nodes for $Q_{n}$. The conditionally $t$-diagnosable network systems are a lass of typical nonlinear systems, in which the state (syndrome) of a node impacted these nodes in its surrounding area. Recent years, there are some studies to analyze nonlinear systems by using online policy iterative optimization algorithms [20, 26]. The combination of these algorithms and our algorithm will be a try to obtain an optimal fault set for considered conditionally $t$-diagnosable network system; this is one of our studies in the future.

\section{Data Availability}

The data used to support the findings of this study are included within the article.

\section{Conflicts of Interest}

The authors declare that there are no conflicts of interest regarding the publication of this paper. 


\section{Acknowledgments}

This work was supported in part by the Natural Science Foundation of China under Grant nos. 61862003 and 61761006 and Natural Science Foundation of the Guangxi Zhuang Autonomous Region of China under Grant no. 2018GXNSFDA281052.

\section{References}

[1] F. P. Preparata, G. Metze, and R. T. Chien, "On the connection assignment problem of diagnosable systems," IEEE Transactions on Electronic Computers, vol. 16, no. 6, pp. 848-854, 1967.

[2] X. Li, J. Fan, C.-K. Lin, and X. Jia, "Diagnosability evaluation of the data center network DCell," The Computer Journal, vol. 61, no. 1, pp. 129-143, 2018.

[3] J. Fan and X. Jia, "Edge-pancyclicity and path-embeddability of bijective connection graphs," Information Sciences, vol. 178, no. 2, pp. 340-351, 2008.

[4] J. Fan, X. Jia, X. Liu, S. Zhang, and J. Yu, "Efficient unicast in bijective connection networks with the restricted faulty node set," Information Sciences, vol. 181, no. 11, pp. 2303-2315, 2011.

[5] S. He, H. Fang, M. Zhang, F. Liu, and Z. Ding, "Adaptive optimal control for a class of nonlinear systems: the online policy iteration approach," IEEE Transactions on Neural Networks and Learning Systems, vol. 31, no. 2, pp. 549-558, 2020.

[6] J. Liang and Q. Zhang, "The $t / s$-diagnosability of hypercube networks under the PMC and comparison models," IEEE Access, vol. 5, no. 1, pp. 5340-5346, 2017.

[7] L.-C. Ye, J.-R. Liang, and H.-X. Lin, "A fast pessimistic diagnosis algorithm for hypercube-like networks under the comparison model," IEEE Transactions on Computers, vol. 65, no. 9, pp. 2884-2888, 2016.

[8] M. Xie, J. Liang, and X. Xiong, "The strong local diagnosability of a hypercube network with missing edges," Complexity, vol. 2018, Article ID 5745628, 10 pages, 2018.

[9] J. Liang, Q. Zhang, and H. Li, "Structural properties and $t / s$ diagnosis for star networks based on the PMC model," IEEE Access, vol. 5, no. 11, pp. 26175-26183, 2017.

[10] W. Yang, H. Lin, and C. Qin, "On the $t / k$-diagnosability of BC networks," Applied Mathematics and Computation, vol. 225, no. 12, pp. 366-371, 2013.

[11] W. Yang, H. Li, and X. Guo, "A kind of conditional fault tolerance of $(n, k)$-star graphs," Information Processing Letters, vol. 110, no. 22, pp. 1007-1011, 2010.

[12] X. Yang, "A fast diagnosis algorithm for hypercube multiprocessor systems under the pessimistic one-step diagnosis," Journal of Parallel and Distributed Computing, vol. 64, no. 3, pp. 537-553, 2004

[13] D.-R. Duh, C.-H. Chen, and K.-N. Chang, "A fast pessimistic diagnosis algorithm for generalized hypercube multicomputer systems," The Journal of Supercomputing, vol. 61, no. 3, pp. 605-618, 2012.

[14] B. Cheng, J. Fan, X. Jia, and J. Jia, "Parallel construction of independent spanning trees and an application in diagnosis on Möbius cubes," The Journal of Supercomputing, vol. 65, no. 3, pp. 1279-1301, 2013.

[15] L. Xu, S. Zhou, and G. Lian, "Conditional diagnosability of multiprocessor systems based on complete-transposition graphs," Discrete Applied Mathematics, vol. 247, pp. 367-379, 2018.
[16] S. He, M. Zhang, H. Fang, F. Liu, X. Luan, and Z. Ding, "Adaptive optimization and reinforcement learning of stochastic Markov jump systems with completely unknown dynamic information," Neural Computing and Applications, vol. 31, 2019.

[17] W. Liu, J. Fan, X. Jia, and S. Zhang, "The spined cube: a new hypercube variant with smaller diameter," Information Processing Letters, vol. 111, no. 12, pp. 561-567, 2011.

[18] G.-H. Hsu and J. J. M. Tan, "A local diagnosability measure for multiprocessor systems," IEEE Transactions on Parallel and Distributed Systems, vol. 18, no. 5, pp. 598-607, 2007.

[19] P. L. Lai, J. J. M. Tan, C. P. Chang, and L. H. Hsu, "Conditional diagnosability measures for large multiprocessor systems," IEEE Transactions on Computers, vol. 54, no. 2, pp. 165-175, 2005.

[20] S. He, H. Fang, M. Zhang, F. Liu, X. Luan, and Z. Ding, "Online policy iterative-based $H_{\infty}$ optimization algorithm for a class of nonlinear systems," Information Sciences, vol. 495, pp. 1-13, 2019.

[21] J. Zheng, S. Latifi, E. Regentova, K. Luo, and X. Wu, "Diagnosability of star graphs under the comparison diagnosis model," Information Processing Letters, vol. 93, no. 1, pp. 29-36, 2005.

[22] S. Zhou, S. Song, X. Yang, and L. Chen, "On conditional fault tolerance and diagnosability of hierarchical cubic networks," Theoretical Computer Science, vol. 609, no. 9, pp. 421-433, 2016.

[23] S. Chen, L. Lin, L. Xu, and D. Wang, “The $t / k$-diagnosability of star graph networks," IEEE Transactions on Computers, vol. 62, no. 2, pp. 547-555, 2015.

[24] X. Wang, D. J. Evans, and G. M. Megson, "On the maximal connected component of a hypercube with faulty vertices III," International Journal of Computer Mathematics, vol. 83, no. 1, pp. 27-37, 2006.

[25] L. H. Hsu, E. Cheng, L. Lipták, J. J. M. Tan, C.-K. Lin, and T.-Y. Ho, "Component connectivity of the hypercubes," International Journal of Computer Mathematics, vol. 89, no. 2, pp. 137-145, 2012.

[26] D. Liu and Q. Wei, "Policy iteration adaptive dynamic programming algorithm for discrete-time nonlinear systems," IEEE Transactions on Neural Networks and Learning Systems, vol. 25, no. 3, pp. 621-634, 2014. 